\title{
THERMODYNAMIC PROPERTIES AND STABILITY FOR THE HEAT FLUX EQUATION WITH LINEAR MEMORY
}

\author{
BY \\ C. GIORGI (Dip. di Matematica, Univ. della Calabria, Arcavacata di Rende (CS), Italy) \\ AND \\ G. GENTILI (Dip. di Matematica, Università di Bologna, Italy)
}

\begin{abstract}
Within the linearized theory of heat conduction with fading memory, some restrictions on the constitutive equations are found as a direct consequence of thermodynamic principles. Such restrictions allow us to obtain existence, uniqueness, and stability results for the solution to the heat flux equation. Both problems, which respectively occur when the instantaneous conductivity $k_{0}$ is positive or vanishes, are considered.
\end{abstract}

1. Introduction. This paper deals with the asymptotic behaviour of the solution of the heat flux equation with memory. We restrict our attention to a homogeneous and isotropic rigid heat conductor with linear memory occupying a fixed bounded domain $\Omega \subset \mathbb{R}^{3}$ with smooth boundary $\partial \Omega$. Moreover, if we consider only small variations of the temperature $\theta(\mathbf{x}, t)$ from a reference value $\theta_{0}$ and small temperature gradients $\nabla \theta(\mathbf{x}, t)$ we may assume that the internal energy $\varepsilon(\mathbf{x}, t)$ and heat flux $\mathbf{q}(\mathbf{x}, t)$ are described by the following linearized constitutive equations:

$$
\begin{aligned}
& \varepsilon(\mathbf{x}, t)=\varepsilon_{0}+\alpha_{0} u(\mathbf{x}, t)+\int_{0}^{\infty} \alpha^{\prime}(s) u^{t}(\mathbf{x}, s) d s, \\
& \mathbf{q}(\mathbf{x}, t)=-k_{0} \nabla u(\mathbf{x}, t)-\int_{0}^{\infty} k^{\prime}(s) \nabla u^{t}(\mathbf{x}, s) d s,
\end{aligned}
$$

where $u(\mathbf{x}, t)=\theta(\mathbf{x}, t)-\theta_{0}$ is the temperature variation field, and $u^{t}(\mathbf{x}, s)=$ $u(\mathbf{x}, t-s)$.

As usual in material with memory, a fading memory principle for the relaxation functions $\alpha^{\prime}$ and $k^{\prime}$ is required. Following Day's argument [8] we may state the principle in a weak form, which is equivalent to

$$
\alpha^{\prime}, k^{\prime} \in L^{1}\left(\mathbb{R}^{+}\right) \text {. }
$$

Hence, the heat capacity and thermal conductivity are defined respectively by

$$
\alpha(t)=\alpha_{0}+\int_{0}^{t} \alpha^{\prime}(s) d s, \quad k(t)=k_{0}+\int_{0}^{t} k^{\prime}(s) d s,
$$

Received August 5, 1991.

1991 Mathematics Subject Classification. Primary 80A20, 45K05. 
and the values $\alpha_{\infty}=\lim _{t \rightarrow \infty} \alpha(t)$ and $k_{\infty}=\lim _{t \rightarrow \infty} k(t)$ represent respectively the equilibrium heat capacity and the equilibrium thermal conductivity.

Moreover, we impose the a priori condition

$$
\alpha_{0}>0,
$$

which follows from the physical evidence that, if the temperature of a body is constant up to a certain time $t=0$ and increases instantaneously at $t=0$, then the internal energy of the body increases too.

The ensuing evolution problem with mixed boundary conditions and initial history data is given by

$$
\begin{array}{ll}
\dot{\varepsilon}(\mathbf{x}, t)+\nabla \cdot \mathbf{q}(\mathbf{x}, t)=r(\mathbf{x}, t) & \text { on } \Omega \times \mathbb{R}^{+}, \\
\mathbf{q}(\mathbf{x}, t) \cdot \mathbf{n}(\mathbf{x})=\phi(\mathbf{x}, t) & \text { on } \partial \Omega_{q} \times \mathbb{R}^{+}, \\
\theta(\mathbf{x}, t)=\theta_{0} & \text { on } \partial \Omega_{u} \times \mathbb{R}^{+}, \\
\theta(\mathbf{x}, t)=\theta_{0}+u_{0}(\mathbf{x}, t) & \text { on } \Omega \times \mathbb{R}^{-},
\end{array}
$$

where $r, \phi$, and $u_{0}$ represent respectively the heat source, the heat flux on the boundary $\partial \Omega_{q}$, and the temperature variation field up to the time $t=0$, while $\Omega$ is a bounded domain with boundary $\partial \Omega=\partial \Omega_{u} \cup \partial \Omega_{q}\left(\partial \Omega_{u} \cap \partial \Omega_{q}=\varnothing\right)$ and $\mathbf{n}(\mathbf{x})$ is the outward normal on $\partial \Omega_{q}$.

Problems such as (1.5) have been studied by several authors. For instance, Nunziato [7] and Grabmueller [19] proved existence and uniqueness for generalized solutions; Miller [11] has studied stability and continuous dependence on parameter.

This paper differs from the previous ones in its investigation of the connection between the complete set of thermodynamic restrictions on the relaxation functions, $\alpha$ and $k$, and the solvability and stability of the problem (1.1), (1.5). In essence we prove that the fading-memory principle, stated by (1.2), condition (1.4), and the Second Law of Thermodynamics, in the form of the Clausius property, are sufficient conditions to obtain existence, uniqueness, and stability for the solution. Finally, we stress that a Neumann boundary condition involving the heat flux, instead of the temperature gradient flux, is considered. Such conditions, in fact, are not completely equivalent in materials that satisfy $(1.1)_{2}$.

The complete set of restrictions imposed on the heat capacity and thermal conducivity by the Second Law of Thermodynamics is derived in Sec. 2. It is interesting to compare such results (especially Corollaries 2.1 and 2.3) with the set of assumptions required by Miller to prove existence, uniqueness, and stability in [11]. In fact all these sufficient conditions turn out to be thermodynamic consequences. In order to study the evolution problem (1.5), we discuss separately the cases $k_{0}>0$ and $k_{0}=0$. In effect, these cases are quite different because the positiveness of $k_{0}$ leads to a parabolic problem, whereas when $k_{0}$ vanishes the problem might become hyperbolic.

The parabolic problem, when $k_{0}>0$, is considered in Sec. 3. Existence, uniqueness, and asymptotic behaviour of the solution are proved, and a stability result is given. Moreover, under suitable regularity hypotheses on the data, the solution turns out to be asymptotically stable. Similar results are obtained when $k_{0}=0$ in Sec. 4 , 
whereas in Sec. 5 we discuss the meaning of some functional spaces and restrictions on the initial-history data introduced in the previous section.

As a concluding remark we emphasize that weaker conditions than those of Miller are needed here, and nevertheless more accurate stability results are obtained when $k_{0}$ is positive or vanishes. In particular, our assumptions allow $k^{\prime}(t)$ to have an integrable singularity at $t=0$ whereas Miller implicitly assumes that $k^{\prime}(0)$ is finite. More importantly, in the case $k_{0}=0$ Miller's assumption $\left(\mathrm{S}^{\prime}\right)$ (see [11, p. 325]) fails to be valid, as proved by Corollary 2.3 , so that no stability property follows from his argument. Thus, in that case, our stability results are new.

Next we give some notation for Laplace and Fourier transforms and convolution. For later convenience we denote by $\hat{f}$ the (formal) Fourier transform of any scalaror vector-valued function $f$ defined on $\Omega \times \mathbb{R}$, namely

$$
\hat{f}(\mathbf{x}, \omega)=\int_{-\infty}^{\infty} f(\mathbf{x}, s) \exp (-i \omega s) d s, \quad(\mathbf{x}, \omega) \in \Omega \times \mathbb{R} .
$$

Similarly, letting the subscripts $\mathbf{s}$ (c) denote the half-range Fourier sine (cosine) transform, for any function $g$ defined on $\Omega \times \mathbb{R}^{+}$we have (formally)

$$
\hat{g}_{\mathrm{c}}(\mathbf{x}, \omega)=\int_{0}^{\infty} g(\mathbf{x}, s) \cos \omega s d s, \quad \hat{g}_{\mathrm{s}}(\mathbf{x}, \omega)=\int_{0}^{\infty} g(\mathbf{x}, s) \sin \omega s d s .
$$

If $g(\mathbf{x}, \cdot)$ belongs to $V$, where $V=L^{1}\left(\mathbb{R}^{+}\right)$or $V=L^{2}\left(\mathbb{R}^{+}\right)$, then its Laplace transform, defined by

$$
\tilde{g}(\mathbf{x}, p)=\int_{0}^{\infty} g(\mathbf{x}, s) \exp (-p s) d s, \quad(\mathbf{x}, p) \in \Omega \times \mathbb{C}^{+}
$$

$\left(\mathbb{C}^{+}=\{p \in \mathbb{C} ; \operatorname{Re}(p) \geq 0\}\right)$, is analytic on $\mathbb{C}^{++}=\{p \in \mathbb{C} ; \operatorname{Re}(p)>0\}$. Unless otherwise stated, we identify a function $g$ with its causal extension to $\mathbb{R}$, i.e., $g(\mathbf{x}, t)=0$ when $t<0$. In this way the Laplace transform $\tilde{g}(\mathbf{x}, p)$, when $p=i \omega$, can be considered as the Fourier transform, i.e., $\tilde{g}(\mathbf{x}, i \omega)=\hat{g}(\mathbf{x}, \omega)$. Moreover, we have

$$
\hat{g}(\mathbf{x}, \omega)=\hat{g}_{\mathrm{c}}(\mathbf{x}, \omega)-i \hat{g}_{\mathrm{s}}(\mathbf{x}, \omega) .
$$

Finally, given any pair $(f, g)$ of scalar- or vector-valued functions defined on $\Omega \times \mathbb{R}$, let “*” and “ $*_{\mathrm{F}}$ " denote respectively the Laplace and Fourier time-convolution products, namely

$$
f * g(t)=\int_{0}^{t} f(s) g(t-s) d s \text { and } \quad f_{*_{\mathrm{F}}} g(t)=\int_{-\infty}^{+\infty} f(s) g(t-s) d s .
$$

2. Thermodynamic restrictions. Let the thermal history $\Lambda^{t}$ be defined as $\Lambda^{t}=$ $\left(u^{t}, \mathbf{g}^{t}\right)$, where $\mathbf{g}^{t}=\nabla u^{t}$ and $u^{t}(s)=u(t-s), s \in \mathbb{R}^{+}$, and let the set of all periodic thermal histories be denoted by $\mathscr{P}$. Then the Second Law of Thermodynamics for rigid heat conductors in the Clausius form is stated as follows (see [15]).

SECOND Law. For each periodic thermal history $\Lambda^{t}=\left(u^{t}, g^{t}\right) \in \mathscr{P}$ with period $d$ and for each $t_{0} \in \mathbb{R}$, the following inequality holds:

$$
\int_{t_{0}}^{t_{0}+d}\left[\frac{\dot{\varepsilon}(\mathbf{x}, t)}{\theta_{0}+u(\mathbf{x}, t)}+\frac{\mathbf{q}(\mathbf{x}, t) \cdot \mathbf{g}(\mathbf{x}, t)}{\left(\theta_{0}+u(\mathbf{x}, t)\right)^{2}}\right] d t \leq 0 .
$$


Under linear approximation (i.e., small variations $u(\mathbf{x}, t)$ with respect to a given reference temperature $\theta_{0}$ and small $\left.\mathbf{g}(\mathbf{x}, t)\right)$, the quantity $\theta^{-1}(\mathbf{x}, t)=\left(\theta_{0}+u(\mathbf{x}, t)\right)^{-1}$ can be substituted by its linear Taylor polynomial, thus yielding the approximate inequality

$$
\theta_{0}^{-2} \int_{t_{0}}^{t_{0}+d}\left[\dot{\varepsilon}(\mathbf{x}, t)\left(\theta_{0}-u(\mathbf{x}, t)\right)+\mathbf{q}(\mathbf{x}, t) \cdot \mathbf{g}(\mathbf{x}, t)\right] d t \leq 0,
$$

where $\varepsilon$ and $\mathbf{q}$ are given by the linearized constitutive equations (1.1). Moreover, because of periodicity, (2.1) is equivalent to

$$
\theta_{0}^{-2} \int_{0}^{d}[\varepsilon(\mathbf{x}, t) \dot{u}(\mathbf{x}, t)+\mathbf{q}(\mathbf{x}, t) \cdot \mathbf{g}(\mathbf{x}, t)] d t \leq 0 .
$$

The complete statement of the Second Law, however, specifies that the inequality refers to an irreversible process, whereas the equality occurs in reversible processes only (see for instance [18]). Actually, for a heat conductor satisfying (1.1) a process is reversible if and only if it holds the temperature constant in time $\left(\theta(t)=\theta_{0}\right)$ and uniform in space $(\mathbf{g}(t)=\mathbf{0})$, so that the ensuing thermal history $\Lambda^{t}=\left(u^{t}, \mathbf{g}^{t}\right) \in \mathscr{P}$ vanishes identically. Thereby, the inequality $(2.2)$ is verified as an equality only if $\dot{u}=0$ and $\mathbf{g}=\mathbf{0}$.

Summarizing and putting (1.1) into (2.2), the Second Law for hereditary rigid heat conductors under linear approximation can be reformulated as follows:

SECOND LAw. Relative to rigid heat conductors satisfying (1.1)-(1.2), for each $d$ periodic thermal history $\Lambda^{t}=\left(u^{t}, \mathbf{g}^{t}\right) \in \mathscr{P}$ not vanishing identically, the following strict inequality holds :

$$
\begin{aligned}
\int_{0}^{d}\left[\int_{0}^{\infty} \alpha^{\prime}(s) u^{t}(\mathbf{x}, s) \dot{u}(\mathbf{x}, t) d s-\right. & k_{0} \mathbf{g}(\mathbf{x}, t) \cdot \mathbf{g}(\mathbf{x}, t) \\
& \left.-\int_{0}^{\infty} k^{\prime}(s) \mathbf{g}^{t}(\mathbf{x}, s) \cdot \mathbf{g}(\mathbf{x}, t) d s\right] d t<0 .
\end{aligned}
$$

In order to exploit the consequences of this statement we consider a thermal history $\Lambda^{t}$ generated by the function

$$
\Lambda(\mathbf{x}, t)=\Lambda_{1}(\mathbf{x}) \cos \omega t+\Lambda_{2}(\mathbf{x}) \sin \omega t
$$

with $\Lambda_{i}(\mathbf{x})=\left(u_{i}(\mathbf{x}), \mathbf{g}_{i}(\mathbf{x})\right)$ depending only on the spatial variable. Such a history is periodic with period $d=2 \pi / \omega$.

THEOREM 2.1. For a rigid heat conductor satisfying (1.1)-(1.2), the Second Law of Thermodynamics holds if and only if the relaxation functions $k^{\prime}$ and $\alpha^{\prime}$ satisfy:

$$
\begin{array}{ll}
k_{0}+\hat{k}_{\mathrm{c}}^{\prime}(\omega)>0 & \forall \omega \in \mathbb{R}, \\
\omega \hat{\alpha}_{\mathrm{s}}^{\prime}(\omega)>0 & \forall \omega \neq 0 .
\end{array}
$$

Proof. Substituting $\Lambda(\mathbf{x}, t)$ as expressed by (2.4) into (2.3) and taking into account that

$$
\begin{gathered}
\Lambda^{t}(\mathbf{x}, s) \equiv \Lambda(\mathbf{x}, t-s)=\Lambda(\mathbf{x}, t) \cos \omega s-\omega^{-1} \dot{\Lambda}(\mathbf{x}, t) \sin \omega s \\
\dot{\Lambda}(\mathbf{x}, t)=\omega \Lambda_{2}(\mathbf{x}) \cos \omega s-\omega \Lambda_{1}(\mathbf{x}) \sin \omega s
\end{gathered}
$$


it follows that

$$
\begin{aligned}
\int_{0}^{d}\left[\hat{\alpha}_{\mathrm{c}}^{\prime}(\omega) u(\mathbf{x}, t) \dot{u}(\mathbf{x}, t)-\frac{1}{\omega} \hat{\alpha}_{\mathrm{s}}^{\prime}(\omega) \dot{u}(\mathbf{x}, t)^{2}\right. \\
\left.\quad-\left(k_{0}+\hat{k}_{\mathrm{c}}^{\prime}(\omega)\right)|\mathbf{g}(\mathbf{x}, t)|^{2}+\frac{1}{\omega} \hat{k}_{\mathrm{s}}^{\prime}(\omega) \mathbf{g}(\mathbf{x}, t) \cdot \dot{\mathbf{g}}(\mathbf{x}, t)\right] d t \\
=-\int_{0}^{d}\left[\frac{1}{\omega} \hat{\alpha}_{\mathrm{s}}^{\prime}(\omega) \dot{u}(\mathbf{x}, t)^{2}+\left(k_{0}+\hat{k}_{\mathrm{c}}^{\prime}(\omega)\right)|\mathbf{g}(\mathbf{x}, t)|^{2}\right] d t<0 .
\end{aligned}
$$

Now if $\Lambda_{1}=(0, \mathbf{g})$ and $\Lambda_{2}=(u, \mathbf{0})$ from $(2.7)$ we have

$$
\begin{array}{r}
\int_{0}^{d}\left[\omega \hat{\alpha}_{\mathrm{s}}^{\prime}(\omega) u^{2} \cos ^{2} \omega t+\left(k_{0}+\hat{k}_{\mathrm{c}}^{\prime}(\omega)\right)|\mathbf{g}|^{2} \cos ^{2} \omega t\right] d t \\
=\frac{d}{2}\left[\omega \hat{\alpha}_{\mathrm{s}}^{\prime}(\omega) u^{2}+\left(k_{0}+\hat{k}_{\mathrm{c}}^{\prime}(\omega)\right)|\mathbf{g}|^{2}\right]>0 \quad \forall \omega \in \mathbb{R}
\end{array}
$$

and by the arbitrariness of $u$ and $g$ relations (2.5)-(2.6) follow.

Paralleling the argument explained in [15], it is easily shown that (2.5)-(2.6) are also sufficient conditions for the validity of the Second Law for rigid heat conductors in the form (2.3).

As a consequence of Theorem 2.1 we have the following corollaries.

Corollary 2.1. For a rigid heat conductor satisfying (1.1)-(1.2), if $\alpha_{0}>0$ the Second Law of Thermodynamics yields

$$
\begin{array}{ll}
\operatorname{Re}\left\{k_{0}+\tilde{k}^{\prime}(p)\right\}>0 & \forall p \in \mathbb{C}^{+}, \\
\operatorname{Re}\left\{p\left[\alpha_{0}+\tilde{\alpha}^{\prime}(p)\right]\right\}>0 & \forall p \in \mathbb{C}^{+} \backslash\{0\} .
\end{array}
$$

Proof. We first observe that the left-hand sides of (2.8) and (2.9) are real parts of analytic functions on $\mathbb{C}^{++}$. Then, by using the Hilbert integral representation for Laplace transforms, that is,

$$
\tilde{f}(p)=\frac{2}{\pi} \int_{0}^{\infty} \frac{\omega}{p^{2}+\omega^{2}} \hat{f}_{\mathrm{s}}(\omega) d \omega, \quad \tilde{f}(p)=\frac{2}{\pi} \int_{0}^{\infty} \frac{p}{p^{2}+\omega^{2}} \hat{f}_{\mathrm{c}}(\omega) d \omega, \quad p \in \mathbb{C}^{++},
$$

we have

$$
\begin{aligned}
\operatorname{Re}\left\{p \tilde{\alpha}^{\prime}(p)\right\} & =\frac{2}{\pi} \int_{0}^{\infty} \omega \hat{\alpha}_{\mathrm{s}}^{\prime}(\omega)\left(p_{1}^{2}+p_{2}^{2}+\omega^{2}\right) \varphi\left(p_{1}, p_{2}, \omega\right) d \omega \\
\operatorname{Re}\left\{k_{0}+\tilde{k}^{\prime}(p)\right\} & =\frac{2}{\pi} \int_{0}^{\infty}\left(k_{0}+\hat{k}_{\mathrm{c}}^{\prime}(\omega)\right)\left(p_{1}^{2}+p_{2}^{2}+\omega^{2}\right) \varphi\left(p_{1}, p_{2}, \omega\right) d \omega
\end{aligned}
$$

where $p=p_{1}+i p_{2}$ and $\varphi\left(p_{1}, p_{2}, \omega\right) d \omega=p_{1}\left(\left(p_{1}^{2}-p_{2}^{2}+\omega^{2}\right)^{2}+4 p_{1}^{2} p_{2}^{2}\right)^{-1} \quad\left(p_{1}>0\right)$. Because of (2.5) -(2.6) both of the integrals (2.10) and (2.11) are positive. Then, since $\alpha_{0}>0$ and $\operatorname{Re} p>0,(2.8)$ and (2.9) follow.

Corollary 2.2. Under previous assumptions, the Second Law leads to the following properties :

$$
\text { (a) } \alpha_{0}<\alpha(t)<2 \alpha_{\infty}-\alpha_{0}
$$

and moreover, if $\alpha^{\prime}(0)$ exists, it must be nonnegative;

$$
\text { (b) } k_{0} \geq 0 \text { and } k_{\infty}>0 \text {. }
$$


Proof. From the inversion formula

$$
\alpha^{\prime}(t)=\frac{2}{\pi} \int_{0}^{\infty} \hat{\alpha}_{\mathrm{s}}^{\prime}(\omega) \sin \omega t d \omega \quad \text { a.e. on } \mathbb{R}^{+}
$$

and (2.6) it follows that

$$
\alpha(t)-\alpha_{0}=\frac{2}{\pi} \int_{0}^{\infty} \omega^{-1}(1-\cos \omega t) \hat{\alpha}_{\mathrm{s}}^{\prime}(\omega) d \omega>0
$$

and, moreover,

$$
\alpha_{\infty}-\alpha_{0}=\frac{2}{\pi} \int_{0}^{\infty} \omega^{-1} \hat{\alpha}_{\mathrm{s}}^{\prime}(\omega) d \omega>0 .
$$

Combining (2.14) and (2.15) we obtain

$$
\alpha(t)-\alpha_{\infty}=\frac{2}{\pi} \int_{0}^{\infty}-\omega^{-1} \cos \omega s \hat{\alpha}_{\mathrm{s}}^{\prime}(\omega) d \omega<\frac{2}{\pi} \int_{0}^{\infty} \omega^{-1} \hat{\alpha}_{\mathrm{s}}^{\prime}(\omega) d \omega=\alpha_{\infty}-\alpha_{0}
$$

so that (2.12) follows. Finally, if $\alpha^{\prime}(0)$ exists by (2.14) it must be nonnegative.

Furthermore, by taking the limit as $\omega \rightarrow \infty$ in (2.5) we obtain (2.13) , whereas taking $\omega=0$ there we obtain

$$
k_{0}+\hat{k}_{\mathrm{c}}^{\prime}(0)=k_{\infty}>0 .
$$

Corollary 2.3. Under the previous assumptions, if $k_{0}=0$ and $k^{\prime}(0)$ does exist, then, as a thermodynamic consequence, we have

$$
k^{\prime}(0)>0 \text { and }\left|k^{\prime}(t)\right|<k^{\prime}(0) \text { a.e. on } \mathbb{R}^{+} .
$$

Moreover, if $k^{\prime \prime}(t)$ has Laplace transform on $\mathbb{C}^{++}$, we have

$$
k_{0}^{\prime}+\tilde{k}^{\prime \prime}(p) \neq 0 \quad \forall p \in \mathbb{C}^{+} \backslash\{0\} .
$$

Proof. When $k_{0}=0$ and $k^{\prime}(0)$ exists, in the sense that $\lim _{t \rightarrow 0^{+}} k^{\prime}(t)=k^{\prime}(0) \in \mathbb{R}$, then the inversion formula

$$
k^{\prime}(t)=\frac{2}{\pi} \int_{0}^{\infty} \hat{k}_{\mathrm{c}}^{\prime}(\omega) \cos \omega t d \omega \quad \text { a.e. on } \mathbb{R}^{+}
$$

and inequality (2.5) lead to

$$
k^{\prime}(0)=\frac{2}{\pi} \int_{0}^{\infty} \hat{k}_{\mathrm{c}}^{\prime}(\omega) d \omega>0 .
$$

Inequalities (2.16) follow from (2.18) and (2.19).

On the other hand, well-known properties of the Laplace transform lead to

$$
k^{\prime}(0)+\tilde{k}^{\prime \prime}(p)=p \tilde{k}^{\prime}(p), \quad p=p_{1}+i p_{2} \in \mathbb{C}^{+} .
$$

First observing that $\tilde{k}^{\prime}(0)=\int_{0}^{\infty} k^{\prime}(s) d s=k_{\infty}<\infty$, from (2.20) it easily follows that

$$
k^{\prime}(0)+\tilde{k}^{\prime \prime}(p)=0 \quad \text { when } p=0 .
$$

Moreover, if $p=i p_{2}$ then $p \tilde{k}^{\prime}(p)=p_{2}\left(\hat{k}_{\mathrm{s}}^{\prime}\left(p_{2}\right)+i \hat{k}_{\mathrm{c}}^{\prime}\left(p_{2}\right)\right)$, so $(2.5),(2.20)$, and $k_{0}=0$ yield

$$
k^{\prime}(0)+\tilde{k}^{\prime \prime}(p) \neq 0 \quad \text { when } p_{1}=0 \text { and } p_{2} \neq 0 .
$$


Applying once again the Hilbert integral representation for Laplace transforms we obtain

$$
\operatorname{Im}\left\{p \tilde{k}^{\prime}(p)\right\}=\frac{4}{\pi} \int_{0}^{\infty} p_{2} \omega^{2} \hat{k}_{\mathrm{c}}^{\prime}(\omega) \varphi\left(p_{1}, p_{2}, \omega\right) d \omega .
$$

Thus, by (2.5) with $k_{0}=0$ and (2.20), it follows that

$$
k^{\prime}(0)+\tilde{k}^{\prime \prime}(p) \neq 0 \quad \text { when } p_{1}>0 \text { and } p_{2} \neq 0 .
$$

Finally, if $p=p_{1}>0$ then (2.18) and (2.5), with $k_{0}=0$, lead to the inequality

$$
p \tilde{k}^{\prime}(p)=p_{1} \int_{0}^{\infty} \exp \left(-p_{1} t\right) k^{\prime}(t) d t=\frac{2}{\pi} \int_{0}^{\infty} p_{1}^{2}\left(p_{1}^{2}+\omega^{2}\right)^{-1} \hat{k}_{\mathrm{c}}^{\prime}(\omega) d \omega>0
$$

so that from (2.20) it follows that

$$
k^{\prime}(0)+\tilde{k}^{\prime \prime}(p) \neq 0 \quad \text { when } p_{1}>0 \text { and } p_{2}=0 .
$$

The collection (2.21)-(2.24) proves (2.17).

Note that the conditions required by Miller (see [11]) to ensure the existence, uniqueness, and stability of solutions to the heat flux equation when $k_{0} \neq 0$ are exactly (2.8)-(2.13). Thus Theorem 2.1 and the ensuing corollaries allow us to give a physical interpretation of these conditions; that is, the constitutive equations of the model need to be compatible with thermodynamics. In the case $k_{0}=0$, however, in order to obtain stability results Miller needs a stronger condition for $k(t)$ which fails to be valid. In essence (see assumption $\left(S^{\prime}\right)$, p. 325 in [11]), he requires that $k_{0}^{\prime}+\tilde{k}^{\prime \prime}(p) \neq 0 \quad \forall p \in \mathbb{C}^{+}$. Unfortunately, it is apparent from (2.21) that this cannot occur if $p=0$ and $k^{\prime} \in L^{1}\left(\mathbb{R}^{+}\right)$; so no stability property follows from his argument when $k_{0}$ vanishes.

3. Existence, uniqueness, and stability results when $k_{0}$ is positive. Substituting the constitutive equations (1.1) into problem (1.5) one obtains the following initialhistory boundary value problem:

$$
\begin{array}{ll}
\alpha_{0} \frac{\partial}{\partial t} u(\mathbf{x}, t)+\int_{0}^{\infty} \alpha^{\prime}(s) \frac{\partial}{\partial t} u^{t}(\mathbf{x}, s) d s-k_{0} \Delta u(\mathbf{x}, t) & \\
\quad-\int_{0}^{\infty} k^{\prime}(s) \Delta u^{t}(\mathbf{x}, s) d s=r(\mathbf{x}, t) & \text { on } \Omega \times \mathbb{R}^{+}, \\
{\left[k_{0} \nabla u(\mathbf{x}, t)+\int_{0}^{\infty} k^{\prime}(s) \nabla u^{t}(\mathbf{x}, s) d s\right] \cdot \mathbf{n}(\mathbf{x})=-\phi(\mathbf{x}, t)} & \text { on } \partial \Omega_{q} \times \mathbb{R}^{+}, \\
u(\mathbf{x}, t)=0 & \text { on } \partial \Omega_{u} \times \mathbb{R}^{+}, \\
u(\mathbf{x}, t)=u_{0}(\mathbf{x}, t) & \text { on } \Omega \times \mathbb{R}^{-},
\end{array}
$$

where $\partial \Omega$ is smooth and constituted by the union of $\partial \Omega_{q}$ and $\partial \Omega_{u}$. We exclude the case $\partial \Omega=\partial \Omega_{q}$, when no temperature is assigned at the boundary. Then, by setting

$$
\begin{gathered}
\bar{u}_{0}(\mathbf{x})=u_{0}(\mathbf{x}, 0), \quad \mathbf{Q}_{0}(\mathbf{x}, t)=\int_{t}^{\infty} k^{\prime}(s) \nabla u_{0}^{t}(\mathbf{x}, s) d s, \\
\dot{U}_{0}(\mathbf{x}, t)=\int_{t}^{\infty} \alpha^{\prime}(s) \frac{\partial}{\partial t} u_{0}^{t}(\mathbf{x}, s) d s, \\
h(\mathbf{x}, t)=r(\mathbf{x}, t)-\dot{U}_{0}(\mathbf{x}, t)+\nabla \cdot \mathbf{Q}_{0}(\mathbf{x}, t), \\
\psi(\mathbf{x}, t)=-\phi(\mathbf{x}, t)-\mathbf{Q}_{0}(\mathbf{x}, t) \cdot \mathbf{n}(\mathbf{x}),
\end{gathered}
$$


the previous problem is replaced by the following equivalent system:

$$
\begin{array}{ll}
\alpha_{0} \frac{\partial}{\partial t} u(\mathbf{x}, t)+\alpha^{\prime} * \frac{\partial}{\partial t} u(\mathbf{x}, t) & \\
\quad-k_{0} \Delta u(\mathbf{x}, t)-k^{\prime} * \Delta u(\mathbf{x}, t)=h(\mathbf{x}, t) & \text { on } \Omega \times \mathbb{R}^{+}, \\
{\left[k_{0} \nabla u(\mathbf{x}, t)+k^{\prime} * \nabla u(\mathbf{x}, t)\right] \cdot \mathbf{n}(\mathbf{x})=\psi(\mathbf{x}, t)} & \text { on } \partial \Omega_{q} \times \mathbb{R}^{+}, \\
u(\mathbf{x}, t)=0 & \text { on } \partial \Omega_{u} \times \mathbb{R}^{+}, \\
u(\mathbf{x}, 0)=\bar{u}_{0}(\mathbf{x}) & \text { in } \Omega .
\end{array}
$$

For later convenience we shall now give some notation for functional spaces, norms, and scalar products. Set $\Omega^{\prime}=\Omega \cup \partial \Omega_{q}$ and define $V_{0}=H_{0}^{1}\left(\Omega^{\prime}\right)$ (see [13]). Let $\|u\|_{1}$ denote the norm in $V_{0},\|u\|$ the norm in $L^{2}(\Omega)$, and $\|u\| \|$ the norm in $L^{2}\left(\mathbb{R}^{+} \times \Omega\right)$. Moreover, from now on we use the following notation:

$$
\langle a, b\rangle=\int_{\Omega} a(\mathbf{x}) b(\mathbf{x}) d \mathbf{x}, \quad\langle a, b\rangle_{q}=\int_{\partial \Omega_{q}} a(\mathbf{x}) b(\mathbf{x}) d \sigma, \quad\|a\|_{q}^{2}=\langle a, a\rangle_{q} .
$$

In order to achieve a stability result relative to problem (3.1) we impose the following assumptions:

(H1) $\bar{u}_{0} \in V_{0}$;

(H2) $r \in L^{2}\left(\mathbb{R}^{+}, L^{2}(\Omega)\right), \phi \in L^{2}\left(\mathbb{R}^{+}, L^{2}\left(\partial \Omega_{q}\right)\right)$;

(H3) $u_{0}(\mathbf{x}, t)$ on $\Omega \times \mathbb{R}^{-}$is such that $\dot{U}_{0} \in L^{2}\left(\mathbb{R}^{+}, L^{2}(\Omega)\right), \mathbf{Q}_{0} \in L^{2}\left(\mathbb{R}^{+}, L^{2}(\Omega)\right)$ and $\nabla \cdot \mathbf{Q}_{0} \in L^{2}\left(\mathbb{R}^{+}, H^{-1}\left(\Omega^{\prime}\right)\right)$

(H4) $u_{0}(\mathbf{x}, t)$ on $\partial \Omega \times \mathbb{R}^{-}$is such that $\mathbf{Q}_{0} \cdot \mathbf{n} \in L^{2}\left(\mathbb{R}^{+}, H^{-1 / 2}\left(\partial \Omega_{q}\right)\right)$.

REMARK 3.1. (H2)-(H4) ensure that

$$
h \in L^{2}\left(\mathbb{R}^{+}, H^{-1}\left(\boldsymbol{\Omega}^{\prime}\right)\right) \quad \text { and } \quad \psi \in L^{2}\left(\mathbb{R}^{+}, H^{-1 / 2}\left(\partial \Omega_{q}\right)\right) .
$$

First consider the Laplace transformed problem of (3.1) which is defined for each $p \in \mathbb{C}^{+}$as

$$
\begin{array}{ll}
p\left[\alpha_{0}+\tilde{\alpha}^{\prime}(p)\right] \tilde{u}(\mathbf{x}, p)-\left[k_{0}+\tilde{k}^{\prime}(p)\right] \Delta \tilde{u}(\mathbf{x}, p)=F(\mathbf{x}, p) & \text { on } \Omega, \\
{\left[k_{0}+\tilde{k}^{\prime}(p)\right] \nabla \tilde{u}(\mathbf{x}, p) \cdot \mathbf{n}(\mathbf{x})=\tilde{\psi}(\mathbf{x}, p)} & \text { on } \partial \Omega_{q}, \\
\tilde{u}(\mathbf{x}, p)=0 & \text { on } \partial \Omega_{u},
\end{array}
$$

where

$$
F(\mathbf{x}, p)=\tilde{h}(\mathbf{x}, p)+\bar{u}_{0}(\mathbf{x})\left[\alpha_{0}+\tilde{\alpha}^{\prime}(p)\right] .
$$

Definition of weak solution. A function $\tilde{u} \in V_{0}$ is called a weak solution of (3.2) if ${ }^{1}$

$$
\int_{\Omega}\left\{p\left[\alpha_{0}+\tilde{\alpha}^{\prime}(p)\right] \tilde{u} \tilde{v}^{*}+\left[k_{0}+\tilde{k}^{\prime}(p)\right] \nabla \tilde{u} \cdot \nabla \tilde{v}^{*}\right\} d x=\int_{\Omega} F \tilde{v}^{*} d x+\int_{\partial \Omega_{q}} \tilde{\psi} \tilde{v}^{*} d x
$$

holds for every $\tilde{v} \in V_{0}$.

${ }^{1} \tilde{v}^{*}$ denotes the complex conjugate of $\tilde{v}$. 
LemMA 3.1. If $\alpha$ and $k$ verify inequalities (2.8)-(2.9) and $u_{0}, \phi$, and $r$ satisfy $(\mathrm{H} 1)-(\mathrm{H} 4)$, then problem $(3.2)$ has one and only one weak solution $\tilde{u}(\cdot, p) \in V_{0}$ for every $p \in \mathbb{C}^{+}$.

Proof. First, note that $\tilde{h}(\cdot, p)$ and $F(\cdot, p)$ are well defined in $H^{-1}(\Omega)$, and $\tilde{\psi}(\cdot, p)$ is well defined in $H^{-1 / 2}\left(\partial \Omega_{q}\right)$ for every $p \in \mathbb{C}^{+}$. Then, by virtue of wellknown theorems on elliptic problems, (3.2) has one and only one weak solution if and only if the operator $L(p)$ defined by

$$
L(p)=-p\left[\alpha_{0}+\tilde{\alpha}^{\prime}(p)\right]+\left[k_{0}+\tilde{k}^{\prime}(p)\right] \Delta
$$

is coercive for every $p \in \mathbb{C}^{+}$, i.e., if and only if the associated bilinear form

$$
a(u, v ; p)=\int_{\Omega}\left\{p\left[\alpha_{0}+\tilde{\alpha}^{\prime}(p)\right] u v^{*}+\left[k_{0}+\tilde{k}^{\prime}(p)\right] \nabla u \cdot \nabla v^{*}\right\} d x
$$

satisfies

$$
|a(u, u ; p)| \geq C(p)\|u\|_{1}^{2} \quad \forall u \in V_{0}, \forall p \in \mathbb{C}^{+}, \text {with } C(p)>0 .
$$

If $p \neq 0$ then

$$
\gamma(p)=\operatorname{Re}\left(p\left[\alpha_{0}+\tilde{\alpha}^{\prime}(p)\right]\right) \operatorname{Re}\left(\left[k_{0}+\tilde{k}^{\prime}(p)\right]\right)\left[\operatorname{Re}\left(p\left[\alpha_{0}+\tilde{\alpha}^{\prime}(p)\right]\right)+\operatorname{Re}\left(\left[k_{0}+\tilde{k}^{\prime}(p)\right]\right)\right]^{-1}
$$

turns out to be positive because of (2.8) and (2.9); thus,

$$
|a(u, u ; p)| \geq|\operatorname{Re}(a(u, u ; p))| \geq \gamma(p)\left(\|u\|^{2}+\|\nabla u\|^{2}\right)=\gamma(p)\|u\|_{1}^{2} \quad \forall p \in \mathbb{C}^{+} \backslash\{0\} .
$$

On the other hand, when $p=0$ we recall that (see [13, Lemma 1.46])

$$
\|\nabla u\| \geq C(\Omega)\|u\|_{1} \quad \forall u \in V_{0},
$$

and from $(2.13)_{2}$ it follows that

$$
|a(u, u ; 0)|=k_{\infty} \int_{\Omega}|\nabla u|^{2} d \mathbf{x} \geq C_{0}\|u\|_{1}^{2} \quad \text { with } C_{0}=k_{\infty} C^{2}(\Omega)>0
$$

and the proof of (3.4) is complete.

We can represent the solution $\tilde{u}$ of (3.2) as follows:

$$
\tilde{u}(\mathbf{x}, p)=\int_{\Omega} \Gamma\left(\mathbf{x}, \mathbf{x}^{\prime} ; p\right) F\left(\mathbf{x}^{\prime}, p\right) d \mathbf{x}^{\prime}+\int_{\partial \Omega} \Gamma\left(\mathbf{x}, \mathbf{x}^{\prime} ; p\right) \tilde{\psi}\left(\mathbf{x}^{\prime}, p\right) d \sigma^{\prime},
$$

where the Green function $\Gamma$ for almost all $\mathbf{x} \in \Omega$ and $p \in \mathbb{C}^{+}$solves the problem

$$
\begin{array}{ll}
p\left[\alpha_{0}+\tilde{\alpha}^{\prime}(p)\right] \Gamma\left(\mathbf{x}, \mathbf{x}^{\prime} ; p\right)-\left[k_{0}+\tilde{k}^{\prime}(p)\right] \Delta^{\prime} \Gamma\left(\mathbf{x}, \mathbf{x}^{\prime} ; p\right)=\delta\left(\mathbf{x}-\mathbf{x}^{\prime}\right) & \mathbf{x}^{\prime} \in \Omega, \\
\nabla^{\prime} \Gamma\left(\mathbf{x}, \mathbf{x}^{\prime} ; p\right) \cdot \mathbf{n}\left(\mathbf{x}^{\prime}\right)=0 & \mathbf{x}^{\prime} \in \partial \Omega_{q}, \\
\Gamma\left(\mathbf{x}, \mathbf{x}^{\prime} ; p\right)=0 & \mathbf{x}^{\prime} \in \partial \Omega_{u} .
\end{array}
$$

Lemma 3.2. Under the hypotheses of Lemma 3.1, for almost all $\mathbf{x} \in \Omega$ and $p \in$ $\mathbb{C}^{+}$problem (3.7) has one and only one solution $\Gamma\left(\mathbf{x}, \mathbf{x}^{\prime} ; p\right)$ with the following properties:

(a) $\Gamma\left(\mathbf{x}, \mathbf{x}^{\prime} ; \cdot\right)$ is continuous on $\mathbb{C}^{+}$;

(b) $\lim _{|p| \rightarrow \infty} \int_{\Omega} p \alpha_{0} \Gamma\left(\mathbf{x}, \mathbf{x}^{\prime} ; p\right) u\left(\mathbf{x}^{\prime}\right) d \mathbf{x}^{\prime}=u(\mathbf{x}) \forall \mu \in C_{0}^{\infty}(r)$;

(c) $\nabla_{x} \Gamma\left(\mathbf{x}, \mathbf{x}^{\prime} ; \cdot\right)$ is continuous on $\mathbb{C}^{+}$; 
(d) $\lim _{|p| \rightarrow \infty} \int_{\Omega} p \alpha_{0} \nabla_{x} \Gamma\left(\mathbf{x}, \mathbf{x}^{\prime} ; p\right) u\left(\mathbf{x}^{\prime}\right) d \mathbf{x}^{\prime}=\nabla u(\mathbf{x}) \forall \mu \in C_{0}^{\infty}(r)$

Proof. (a) follows from the continuity of $a(u, v, \cdot)$ with respect to $p$ (see [10, Lemma 44.1]). Now, multiplying (3.7) ${ }_{1}$ by $\varphi\left(\mathbf{x}^{\prime}\right) \in C_{0}^{\infty}(\Omega)$ and integrating over $\Omega$ we have

$$
\int_{\Omega} p \Gamma\left(\mathbf{x}, \mathbf{x}^{\prime} ; p\right)\left[\left(\alpha_{0}+\tilde{\alpha}^{\prime}(p)\right) \varphi\left(\mathbf{x}^{\prime}\right)-p^{-1}\left(k_{0}+\tilde{k}^{\prime}(p)\right) \Delta \varphi\left(\mathbf{x}^{\prime}\right)\right] d \mathbf{x}^{\prime}=\varphi(\mathbf{x})
$$

so that the limit as $p \rightarrow \infty$ yields

$$
\begin{aligned}
\varphi(\mathbf{x}) & =\lim _{|p| \rightarrow \infty} \int_{\Omega} p \Gamma\left(\mathbf{x}, \mathbf{x}^{\prime} ; p\right)\left[\left(\alpha_{0}+\tilde{\alpha}^{\prime}(p)\right) \varphi\left(\mathbf{x}^{\prime}\right)-p^{-1}\left(k_{0}+\tilde{k}^{\prime}(p)\right) \Delta \varphi\left(\mathbf{x}^{\prime}\right)\right] d \mathbf{x}^{\prime} \\
& =\lim _{|p| \rightarrow \infty} \int_{\Omega} p \alpha_{0} \Gamma\left(\mathbf{x}, \mathbf{x}^{\prime} ; p\right) \varphi\left(\mathbf{x}^{\prime}\right) d \mathbf{x}^{\prime}
\end{aligned}
$$

for every $\varphi \in C_{0}^{\infty}(\Omega)$ and property (b) follows. Finally, by applying the operator $\nabla_{x}$ to $(3.7)_{1}$ we can easily obtain

$$
p\left[\alpha_{0}+\tilde{\alpha}^{\prime}(p)\right] \nabla_{x} \Gamma\left(\mathbf{x}, \mathbf{x}^{\prime} ; p\right)-\left[k_{0}+\tilde{k}^{\prime}(p)\right] \Delta^{\prime} \nabla_{x} \Gamma\left(\mathbf{x}, \mathbf{x}^{\prime} ; p\right)=\delta^{\prime}\left(x-x^{\prime}\right),
$$

so item (c) follows directly from (a), while the proof of (d) parallels that of (b), replacing $(3.7)_{1}$ by $(3.7)_{1}^{\prime}$ and remarking that $\delta^{\prime} \in\left[C_{0}^{\infty}(\Omega)\right]^{\prime}$.

THEOREM 3.1. Under the hypotheses of Lemma 3.1 problem (3.1) has one and only one solution $u \in L^{2}\left(\mathbb{R}^{+}, V_{0}\right)$.

Proof. Since Lemma 3.1 states that the transformed problem (3.2) has one and only one solution $\tilde{u} \in V_{0}$ for every $p \in \mathbb{C}^{+}$, our aim is to prove that, by virtue of Lemma 3.2, this implies the existence and uniqueness of the solution to (3.1). In order to do this, we have to investigate the properties of $\tilde{u}(\mathbf{x}, p)$ when $p=i \omega$. First observe that

$$
\lim _{|p| \rightarrow \infty} F(\mathbf{x}, p)=\alpha_{0} \bar{u}_{0}(\mathbf{x}) \text { a.e. in } \Omega \text { and } \lim _{|p| \rightarrow \infty} \tilde{\psi}(\mathbf{x}, p)=0 \quad \text { a.e. in } \partial \Omega .
$$

Then, from property (b) of Lemma 3.2 it follows that

$$
\begin{aligned}
\lim _{|p| \rightarrow \infty} p \tilde{u}(\mathbf{x}, p)= & \lim _{|p| \rightarrow \infty}\left[\int_{\Omega} p F\left(\mathbf{x}^{\prime}, p\right) \Gamma\left(\mathbf{x}, \mathbf{x}^{\prime} ; p\right) d \mathbf{x}^{\prime}\right. \\
& \left.+\int_{\partial \Omega} p \Gamma\left(\mathbf{x}, \mathbf{x}^{\prime} ; p\right) \tilde{\psi}\left(\mathbf{x}^{\prime}, p\right) d \sigma^{\prime}\right] \\
= & \lim _{|p| \rightarrow \infty} \int_{\Omega} p \Gamma\left(\mathbf{x}, \mathbf{x}^{\prime} ; p\right) \alpha_{0} \bar{u}_{0}\left(\mathbf{x}^{\prime}\right) d \mathbf{x}^{\prime}=\bar{u}_{0}(\mathbf{x}) \text { a.e. in } \Omega
\end{aligned}
$$

and property (d) of Lemma 3.2 leads to

$$
\begin{aligned}
\lim _{|p| \rightarrow \infty} p \nabla_{x} \tilde{u}(\mathbf{x}, p)= & \lim _{|p| \rightarrow \infty}\left[\int_{\Omega} p F(\mathbf{y}, p) \nabla_{x} \Gamma(\mathbf{x}, \mathbf{y} ; p) d \mathbf{y}\right. \\
& \left.+\int_{\partial \Omega} p \nabla_{x} \Gamma(\mathbf{x}, \mathbf{y} ; p) \tilde{\psi}(\mathbf{y}, p) d \sigma_{\mathbf{y}}\right] \\
= & \lim _{|p| \rightarrow \infty} \int_{\Omega} p \nabla_{x} \Gamma(\mathbf{x}, \mathbf{y} ; p) \alpha_{0} \bar{u}_{0}(\mathbf{x}) d \mathbf{y}=\nabla \bar{u}_{0}(\mathbf{x}) \quad \text { a.e. in } \Omega .
\end{aligned}
$$


Setting $p=i \omega$ and observing that that $\tilde{u}(\mathbf{x}, i \omega)=\hat{u}(\mathbf{x}, \omega)$ and $\hat{u} \in L_{\mathrm{loc}}^{2}\left(\mathbb{R}, V_{0}\right)$, by (3.6) and previous lemmas, it follows that $\hat{u} \in L^{2}\left(\mathbb{R}, V_{0}\right)$. Finally, by setting

$$
v(\mathbf{x}, t)= \begin{cases}0, & t<0, \\ u(\mathbf{x}, t), & t \geq 0,\end{cases}
$$

Parseval's Theorem yields

$$
\begin{aligned}
2 \pi^{-1} \int_{-\infty}^{\infty}\left(|\hat{u}(\mathbf{x}, \omega)|^{2}+|\nabla \hat{u}(\mathbf{x}, \omega)|^{2}\right) d \omega & =\int_{-\infty}^{\infty}\left(|v(\mathbf{x}, t)|^{2}+|\nabla v(\mathbf{x}, t)|^{2}\right) d t \\
& =\int_{0}^{\infty}\left(|u(\mathbf{x}, t)|^{2}+|\nabla u(\mathbf{x}, t)|^{2}\right) d t
\end{aligned}
$$

Hence it follows that

$$
\int_{0}^{\infty}\left(\|u(\mathbf{x}, t)\|^{2}+\|\nabla u(\mathbf{x}, t)\|^{2}\right) d t=\frac{1}{2 \pi} \int_{-\infty}^{+\infty}\left(\|\hat{u}(\mathbf{x}, \omega)\|^{2}+\|\nabla \hat{u}(\mathbf{x}, \omega)\|^{2}\right) d t<\infty
$$

and the theorem is so proved.

We now complete the treatment of the parabolic case by showing how the solution is controlled by data. In particular, we are going to get a theorem that may be considered both a control theorem, when sources $r$ and $\phi$ vary but $u_{0}=0 \quad \forall t<0$, and a stability theorem in the usual sense, when $r$ and $\phi$ vanish.

Let $(\mathrm{H} 1)-(\mathrm{H} 4)$ be valid, so that we choose only initial histories belonging to

$$
\mathscr{H}=\left\{u_{0}: \bar{\Omega} \times \mathbb{R}^{-} \rightarrow \mathbb{R} ; u_{0} \text { satisfies }(\mathrm{H} 1),(\mathrm{H} 3) \text {, and }(\mathrm{H} 4)\right\} .
$$

Consider problem (3.1) again and set

$$
f(\mathbf{x}, t)=r(\mathbf{x}, t)-\dot{U}_{0}(\mathbf{x}, t) .
$$

Thus $(3.1)$, can be replaced by

$$
\alpha_{0} \frac{\partial}{\partial t} u(\mathbf{x}, t)+\alpha^{\prime} * \frac{\partial}{\partial t} u(\mathbf{x}, t)-k_{0} \Delta u(\mathbf{x}, t)-k^{\prime} * \Delta u(\mathbf{x}, t)=f(\mathbf{x}, t)+\nabla \cdot \mathbf{Q}_{0}(\mathbf{x}, t) .
$$

Remark 3.2. (H2)-(H3) yield $f \in L^{2}\left(\mathbb{R}^{+}, L^{2}(\Omega)\right)$.

In order to obtain a priori estimates we take the product of (3.8) by $u$ and integrate over $\Omega \times[0, T]$, yielding ${ }^{2}$

$$
\begin{gathered}
\int_{\Omega} \int_{0}^{T}\left[\alpha_{0} \frac{\partial u}{\partial t} u+\left(\alpha^{\prime} * \frac{\partial u}{\partial t}\right) u-\left(k_{0} \Delta u\right) u-\left(k^{\prime} * \Delta u\right) u\right] d \mathbf{x} d t \\
=\int_{\Omega} \int_{0}^{T}\left[f u+\nabla \cdot \mathbf{Q}_{0} u\right] d \mathbf{x} d t \\
\frac{1}{2} \alpha_{0}\left(\|u(T)\|^{2}-\left\|\bar{u}_{0}\right\|^{2}\right)+\int_{0}^{T}\left\langle\alpha^{\prime} * \frac{\partial u}{\partial t}, u\right\rangle d t+\int_{0}^{T}\left[k_{0}\|\nabla u\|^{2}+\left\langle k^{\prime} * \nabla u, \nabla u\right\rangle\right] d t \\
=\int_{0}^{T}\left[\langle f, u\rangle-\left\langle\mathbf{Q}_{0}, \nabla u\right\rangle-\langle\phi, u\rangle_{q}\right] d t .
\end{gathered}
$$

\footnotetext{
${ }^{2}$ In the sequel, for ease of writing, the dependence on $\mathbf{x}$ and $t$ within the integral expressions is understood but not written except for the dependence on $\omega$ in the Fourier transformed functions.
} 
If we extend the solution $u(\mathbf{x}, t)$ as follows,

$$
v_{T}(t)=\left\{\begin{array}{ll}
u(t), & t \in[0, T], \\
0, & t \notin[0, T],
\end{array} \quad \frac{\partial}{\partial t} v_{T}(t)= \begin{cases}\frac{\partial}{\partial t} u(t), & t \in] 0, T[, \\
\delta(t) \bar{u}_{0}, & t=0, \\
-\delta(t-T) u(T), & t=T, \\
0, & t \notin[0, T],\end{cases}\right.
$$

by Parseval's theorem the third term on the left-hand side of (3.9) becomes

$$
\begin{aligned}
\int_{0}^{T}\left[k_{0}\|\nabla u\|^{2}+\left\langle k^{\prime} * \nabla u, \nabla u\right\rangle\right] d t & =\int_{-\infty}^{+\infty}\left[k_{0}\left\|\nabla v_{T}\right\|^{2}+\left\langle k^{\prime} *_{\mathrm{F}} \nabla v_{T}, \nabla v_{T}\right\rangle\right] d t \\
& =\frac{1}{2 \pi} \int_{-\infty}^{+\infty}\left(k_{0}+\hat{k}^{\prime}(\omega)\right)\left\|\nabla \hat{v}_{T}(\omega)\right\|^{2} d \omega \\
& =\frac{1}{\pi} \int_{0}^{+\infty}\left(k_{0}+\hat{k}_{\mathrm{c}}^{\prime}(\omega)\right)\left\|\nabla \hat{v}_{T}(\omega)\right\|^{2} d \omega .
\end{aligned}
$$

Note that the relation (3.11) defines a norm for $\nabla u(\mathbf{x}, t)$ because of (2.5), so

$$
\|\nabla u\|_{k, T}^{2}=\int_{0}^{T}\left[k_{0}\|\nabla u\|^{2}+\left\langle k^{\prime} * \nabla u, \nabla u\right\rangle\right] d t
$$

and with the aid of (3.5) we can define

$$
L_{k}^{2}\left(0, T ; V_{0}\right)=\left\{u:(0, T) \rightarrow V_{0},\|\| \nabla \|_{k, T}^{2}<\infty\right\} .
$$

Now, by assumption $\lim _{|\omega| \rightarrow \infty} k_{0}+\hat{k}_{\mathrm{c}}^{\prime}(\omega)=k_{0}>0$ and by (2.5) we obtain

$$
\gamma=\inf _{\omega \in \mathbb{R}}\left(k_{0}+\hat{k}_{\mathrm{c}}^{\prime}(\omega)\right)>0
$$

which implies

$$
\begin{aligned}
\|\nabla u\|_{k, T}^{2} & =\frac{1}{\pi} \int_{0}^{+\infty}\left(k_{0}+\hat{k}_{\mathrm{c}}^{\prime}(\omega)\right)\left\|\nabla \hat{v}_{T}(\omega)\right\|^{2} d \omega \\
& \geq \frac{\gamma}{\pi} \int_{0}^{+\infty}\left\|\nabla \hat{v}_{T}(\omega)\right\|^{2} d \omega=\gamma \int_{0}^{T}\|\nabla u\|^{2} d t .
\end{aligned}
$$

Since $\left(k_{0}+\hat{k}_{\mathrm{c}}^{\prime}(\omega)\right)$ is bounded and (3.5) holds we can conclude:

REMARK 3.3. If $k_{0}>0$, the norm of $L_{k}^{2}\left(0, T ; V_{0}\right)$ is equivalent to the usual norm of $L^{2}\left(0, T ; V_{0}\right)$.

Applying (3.10) again, the second term of (3.9) becomes

$$
\begin{aligned}
\int_{0}^{T}\left\langle\alpha^{\prime} * \frac{\partial u}{\partial t}, u\right\rangle d t= & \int_{-\infty}^{+\infty}\left\langle\alpha^{\prime} *_{\mathrm{F}} \frac{\partial v_{T}}{\partial t}, v_{T}\right\rangle d t \\
& -\int_{0}^{T}\left\langle\alpha^{\prime} \bar{u}_{0}, u\right\rangle d t+\int_{0}^{T}\left\langle\alpha^{\prime}(t-T) u(T), u\right\rangle d t
\end{aligned}
$$

But $\alpha^{\prime}(t-T)=0$ a.e. in $(0, T)$, so Parseval's theorem yields

$$
\int_{0}^{T}\left\langle\alpha^{\prime} * \frac{\partial u}{\partial t}, u\right\rangle d t=\frac{1}{\pi} \int_{0}^{\infty} \omega \hat{\alpha}_{\mathrm{s}}^{\prime}(\omega)\left\|\hat{v}_{T}(\omega)\right\|^{2} d \omega-\int_{0}^{T} \alpha^{\prime}\left\langle\bar{u}_{0}, u\right\rangle d t
$$


Finally, substituting (3.11) and (3.13) into (3.9) we obtain

$$
\begin{gathered}
\frac{1}{2} \alpha_{0}\|u(T)\|^{2}+\frac{1}{\pi} \int_{0}^{\infty} \omega \hat{\alpha}_{\mathrm{s}}^{\prime}(\omega)\left\|\hat{v}_{T}(\omega)\right\|^{2} d \omega+\frac{1}{\pi} \int_{0}^{\infty}\left(k_{0}+\hat{k}_{\mathrm{c}}^{\prime}(\omega)\right)\left\|\nabla \hat{v}_{T}(\omega)\right\|^{2} d \omega \\
=\frac{1}{2} \alpha_{0}\left\|\bar{u}_{0}\right\|^{2}+\int_{0}^{T}\left[\left\langle\left(f+\alpha^{\prime} \bar{u}_{0}\right), u\right\rangle-\left\langle\mathbf{Q}_{0}, \nabla u\right\rangle-\langle\phi, u\rangle_{q}\right] d t .
\end{gathered}
$$

On the basis of (3.14) we can state the following theorem.

THEOREM 3.2. If the thermal conductivity $k$ and the heat capacity $\alpha$ are such that

(a) $k_{0}>0$ and $\alpha_{0}>0$,

(b) (1.2), (2.5), and (2.6) are satisfied;

then, under assumptions $(\mathrm{H} 1)-(\mathrm{H} 4)$, the problem (3.1) has one and only one solution $u \in L^{\infty}\left(\mathbb{R}^{+}, L^{2}(\Omega)\right) \cap L^{2}\left(\mathbb{R}^{+}, V_{0}\right)$ and, moreover, the corresponding norms of $u$ are bounded by the norms of the data in their respective spaces.

Proof. We have just observed that

$$
\frac{1}{\pi} \int_{0}^{\infty}\left(k_{0}+\hat{k}_{\mathrm{c}}^{\prime}(\omega)\right)\left\|\nabla \hat{v}_{T}(\omega)\right\|^{2} d \omega=\|\nabla u\|_{k, T}^{2}>C_{1} \int_{0}^{T}\|u\|_{1}^{2} d t
$$

and moreover, by virtue of (2.6), we have

$$
\int_{-\infty}^{+\infty} \omega \hat{\alpha}_{\mathbf{s}}^{\prime}(\omega)\left\|\hat{v}_{T}(\omega)\right\|^{2} d \omega>0
$$

so (3.14) leads to

$$
\begin{aligned}
\frac{1}{2} \alpha_{0}\|u(T)\|^{2} & +C_{1} \int_{0}^{T}\|u\|_{1}^{2} d t<\frac{1}{2} \alpha_{0}\left\|\bar{u}_{0}\right\|^{2} \\
& +\int_{0}^{T}\left[\left\langle\left(f+\alpha^{\prime} \bar{u}_{0}\right), u\right\rangle-\left\langle\mathbf{Q}_{0}, \nabla u\right\rangle-\langle\phi, u\rangle_{q}\right] d t
\end{aligned}
$$

Because of $(\mathrm{H} 1)-(\mathrm{H} 4)$ for any positive constant $\varepsilon$ we can set

$$
\begin{gathered}
\left|\left\langle\mathbf{Q}_{0}, \nabla u\right\rangle\right| \leq \frac{\varepsilon}{2}\|\nabla u\|^{2}+\frac{1}{2 \varepsilon}\left\|\mathbf{Q}_{0}\right\|^{2}, \quad|\langle f, u\rangle| \leq \frac{\varepsilon}{2}\|u\|^{2}+\frac{1}{2 \varepsilon}\|f\|^{2}, \\
\left|\langle\phi, u\rangle_{q}\right| \leq \frac{\varepsilon}{2}\|u\|_{q}^{2}+\frac{1}{2 \varepsilon}\|\phi\|_{q}^{2},
\end{gathered}
$$

and by Theorem 3.1 there exists a constant $C_{2}>0$ such that (see [13, Lemma 1.48])

$$
\|u\|_{q}^{2} \leq C_{2}\|\nabla u\|^{2} \text {. }
$$

Thus, if we choose $\varepsilon$ such that $\nu=\left(C_{1}-\varepsilon\left(1+C_{2}\right) / 2\right)>0$, from (3.14) it follows that

$$
\begin{aligned}
\frac{\alpha_{0}}{2}\|u(T)\|^{2}+\nu \int_{0}^{T}\|u\|_{1}^{2} d t \leq & \frac{\alpha_{0}}{2}\left\|\bar{u}_{0}\right\|^{2}+\frac{1}{2 \varepsilon} \int_{0}^{T}\left(\left\|\mathbf{Q}_{0}\right\|^{2}+\|f\|^{2}+\|\phi\|_{q}^{2}\right) d t \\
& +\int_{0}^{T}\left\langle\alpha^{\prime} \bar{u}_{0}, u\right\rangle d t
\end{aligned}
$$


so that

$$
\begin{gathered}
\|u(T)\|^{2} \leq\left\|\bar{u}_{0}\right\|^{2}+\frac{1}{\alpha_{0} \varepsilon} \int_{0}^{T}\left(\left\|\mathbf{Q}_{0}\right\|^{2}+\|f\|^{2}+\|\phi\|_{q}^{2}\right) d t+\frac{2\left\|\bar{u}_{0}\right\|}{\alpha_{0}} \int_{0}^{T}\left|\alpha^{\prime}\right|\|u\| d t, \\
\nu \int_{0}^{T}\|u\|_{1}^{2} d t \leq \frac{\alpha_{0}}{2}\left\|\bar{u}_{0}\right\|^{2}+\frac{1}{2 \varepsilon} \int_{0}^{T}\left(\left\|\mathbf{Q}_{0}\right\|^{2}+\|f\|^{2}+\|\phi\|_{q}^{2}\right) d t+\left\|\bar{u}_{0}\right\| \int_{0}^{T}\left|\alpha^{\prime}\right|\|u\| d t .
\end{gathered}
$$

Applying Gronwall's generalized Lemma (see [17]) the following inequalities hold for any $T \in \mathbb{R}^{+}$:

$$
\begin{gathered}
\|u(T)\| \leq\left(\left\|\bar{u}_{0}\right\|^{2}+\frac{1}{\alpha_{0} \varepsilon} C\left(\mathbf{Q}_{0}, f, \phi\right)\right)^{1 / 2}+\left\|\bar{u}_{0}\right\| \int_{0}^{T} \frac{\left|\alpha^{\prime}\right|}{\alpha_{0}} d t, \\
\nu \int_{0}^{T}\|u\|_{1}^{2} d t \leq \frac{\alpha_{0}}{2}\left\|\bar{u}_{0}\right\|^{2}+\frac{1}{2 \varepsilon} C\left(\mathbf{Q}_{0}, f, \phi\right)+\left\|\bar{u}_{0}\right\| \sup _{t \in[0, T]}\|u(t)\| \int_{0}^{T}\left|\alpha^{\prime}\right| d t,
\end{gathered}
$$

where $C\left(\mathbf{Q}_{0}, f, \phi\right)=\int_{0}^{\infty}\left(\left\|\mathbf{Q}_{0}\right\|^{2}+\|f\|^{2}+\|\phi\|_{q}^{2}\right) d t<\infty$ by assumption. Finally, since $\bar{u}_{0} \in V_{0}$ and $\left|\alpha^{\prime}(t)\right| \in L^{1}\left(\mathbb{R}^{+}\right)$, taking the limit in (3.16) and (3.17) as $T \rightarrow \infty$ the conclusion follows.

Observe that, if the data are suitably regular, (3.1) has a solution belonging to $H^{1}$ with respect to time; therefore Theorem 3.2 yields asymptotic stability.

4. Existence, uniqueness, and stability results when $k_{0}$ vanishes. Assuming $k_{0}=0$, problem (3.1) becomes

$$
\begin{array}{ll}
\alpha_{0} \frac{\partial}{\partial t} u(\mathbf{x}, t)+\alpha^{\prime} * \frac{\partial}{\partial t} u(\mathbf{x}, t)-k^{\prime} * \Delta u(\mathbf{x}, t)=h(\mathbf{x}, t) & \text { on } \Omega \times \mathbb{R}^{+}, \\
k^{\prime} * \nabla u(\mathbf{x}, t) \cdot \mathbf{n}(\mathbf{x})=\psi(\mathbf{x}, t) & \text { on } \partial \Omega_{q} \times \mathbb{R}^{+}, \\
u(\mathbf{x}, t)=0 & \text { on } \partial \Omega_{u} \times \mathbb{R}^{+}, \\
u(\mathbf{x}, 0)=\bar{u}_{0}(\mathbf{x}) & \text { in } \Omega .
\end{array}
$$

REMARK 4.1. In the case $k_{0}=0$ a compatibility condition between the data arises, i.e., $\lim _{t \rightarrow 0^{-}} q(\mathbf{x}, t) \cdot \mathbf{n}(\mathbf{x})=\phi\left(\mathbf{x}, 0^{+}\right)$, and yields the following restriction on the initial histories:

$$
\int_{0}^{\infty} k^{\prime}(s) \nabla u_{0}(\mathbf{x},-s) d s \cdot \mathbf{n}(\mathbf{x})=-\phi(\mathbf{x}, 0) \quad \text { on } \partial \Omega_{q} .
$$

Nevertheless, by using the method of the previous section, with slight modifications we can still obtain existence and uniqueness results. In order to avoid repetitions, we give only the statement of the theorem.

THEOREM 4.1. If $\alpha$ and $k$, when $k_{0}=0$, satisfy inequalities (2.8)-(2.9), and data $u_{0}, \phi$, and $r$ satisfy $(\mathbf{H 1})-(\mathbf{H} 4)$ then $(4.1)$ has one and only one solution $u \in L^{2}\left(\mathbb{R}^{+}, V_{0}\right)$.

On the other hand, investigating how the norm of the solution is controlled by the norms of the data, in the case $k_{0}=0$ we are no longer able to obtain results close to the previous ones. In essence, when $k_{0}>0$ Theorem 3.2 states that the norm of $u$ in $L^{2}\left(\mathbb{R}^{+}, V_{0}\right)$ is controlled by some $L^{2}$-type norms of the data, whereas if $k_{0}=0$ we can hardly control the norm of $u$ relative to $L_{k}^{2}\left(\mathbb{R}^{+}, V_{0}\right)$ and, what 
is more important, by stronger conditions on the data. To clarify this assertion we make some remarks.

Let $L_{1 / k}^{2}\left(\mathbb{R}^{+}, L^{2}(\Omega)\right)$ denote the Banach space defined by the norm

$$
\|f\|_{1 / k}^{2}=\frac{1}{2 \pi} \int_{-\infty}^{+\infty}\left(k_{0}+\hat{k}_{\mathrm{c}}^{\prime}(\omega)\right)^{-1}\|\hat{f}(\omega)\|^{2} d \omega .
$$

REMARK 4.2. The spaces $L_{1 / k}^{2}\left(\mathbb{R}^{+}, L^{2}(\Omega)\right), L_{k}^{2}\left(\mathbb{R}^{+}, L^{2}(\Omega)\right)$, and $L^{2}\left(\mathbb{R}^{+}, L^{2}(\Omega)\right)$ are equivalent when $k_{0}>0$. On the contrary, when $k_{0}$ vanishes they are not equivalent anymore, but their norms are ordered as follows:

$$
\|\| \cdot\left\|_{k}^{2} \leq\right\|\|\cdot\|\left\|^{2} \leq\right\|\|\cdot\|_{1 / k}^{2} \text {. }
$$

In the following section we shall scrutinize $L_{1 / k}^{2}$ in detail.

Some conditions on the data involving norms of the $L_{1 / k}^{2}$-type have to be assumed in order to achieve a stability result in the case $k_{0}=0$. In particular we impose

(H5) $u_{0}(\mathbf{x}, t)$ on $\Omega \times \mathbb{R}^{-}$is such that $\dot{U}_{0}, \mathbf{Q}_{0} \in L_{1 / k}^{2}\left(\mathbb{R}^{+}, L^{2}(\Omega)\right)$,

(H6) $\phi \in L_{1 / k}^{2}\left(\mathbb{R}^{+}, L^{2}\left(\partial \Omega_{q}\right)\right), r \in L_{1 / k}^{2}\left(\mathbb{R}^{+}, L^{2}(\Omega)\right)$

so that $f=r-\dot{U}_{0} \in L_{1 / k}^{2}\left(\mathbb{R}^{+}, L^{2}(\Omega)\right)$. Choose $u_{0}$ varying in the set

$$
\mathscr{H}_{k}=\left\{u_{0}: \Omega \times \mathbb{R}^{-} \rightarrow \mathbb{R} ; u_{0} \text { satisfies (H1), (H4), and (H5) }\right\} .
$$

THEOREM 4.2. If the thermal conductivity $k$ and the heat capacity $\alpha$ are such that

(a) $k_{0}=0, \alpha_{0}>0$,

(b) (1.2), (2.5), and (2.6) are satisfied,

then, under the assumptions $(\mathrm{H} 1)-(\mathrm{H} 6)$, problem $(4.1)$ has one and only one solution $u \in L^{\infty}\left(\mathbb{R}^{+}, L^{2}(\Omega)\right) \cap L^{2}\left(\mathbb{R}^{+}, V_{0}\right)$; nevertheless only the norms of $u$ in the spaces $L^{\infty}\left(\mathbb{R}^{+}, L^{2}(\Omega)\right)$, and $L_{k}^{2}\left(\mathbb{R}^{+}, V_{0}\right)$ are bounded by suitable norms of the data in their respective spaces.

Proof. By working out $(4.1)_{1}$ we are led to the following equation:

$$
\begin{gathered}
\frac{1}{2} \alpha_{0}\left(\|u(T)\|^{2}-\left\|\bar{u}_{0}\right\|^{2}\right)+\int_{0}^{T}\left[\left\langle\alpha^{\prime} * \frac{\partial u}{\partial t}, u\right\rangle+\left\langle k^{\prime} * \nabla u, \nabla u\right\rangle\right] d t \\
=\int_{0}^{T}\left[\langle f, u\rangle-\left\langle\mathbf{Q}_{0}, \nabla u\right\rangle-\langle\phi, u\rangle_{q}\right] d t
\end{gathered}
$$

and after some manipulations

$$
\begin{gathered}
\frac{1}{2} \alpha_{0}\|u(T)\|^{2}+\frac{1}{\pi} \int_{0}^{\infty} \omega \hat{\alpha}_{\mathrm{s}}^{\prime}(\omega)\left\|\hat{v}_{T}(\omega)\right\|^{2} d \omega+\frac{1}{\pi} \int_{0}^{\infty} \hat{k}_{\mathrm{c}}^{\prime}(\omega)\left\|\nabla \hat{v}_{T}(\omega)\right\|^{2} d \omega \\
=\frac{1}{2} \alpha_{0}\left\|\bar{u}_{0}\right\|^{2}+\int_{0}^{T}\left[\left\langle\left(f+\alpha^{\prime} \bar{u}_{0}\right), u\right\rangle-\left\langle\mathbf{Q}_{0}, \nabla u\right\rangle-\langle\phi, u\rangle_{q}\right] d t \\
\frac{\alpha_{0}}{2}\|u(T)\|^{2}+\frac{1}{2}\|\nabla u\|_{k, T}^{2} \leq \frac{1}{2} \alpha_{0}\left\|u_{0}\right\|^{2}+\int_{0}^{T}\left[\left\langle\left(f+\alpha^{\prime} \bar{u}_{0}\right), u\right\rangle-\left\langle\mathbf{Q}_{0}, \nabla u\right\rangle-\langle\phi, u\rangle_{q}\right] d t
\end{gathered}
$$


Note that (H5)-(H6), (3.15), and Poincaré's inequality yield the following: ${ }^{3}$

$$
\begin{aligned}
\left|\int_{0}^{T}\left\langle\mathbf{Q}_{0}, \nabla u\right\rangle d t\right| & =\frac{1}{2 \pi}\left|\int_{-\infty}^{+\infty}\left\langle\hat{\mathbf{Q}}_{0}(\omega), \nabla \hat{v}_{T}(\omega)\right\rangle d t\right| \leq \frac{1}{2 \varepsilon}\left\|\mathbf{Q}_{0}\right\|_{1 / k}^{2}+\frac{\varepsilon}{2}\|\nabla u\|_{k, T}^{2}, \\
\left|\int_{0}^{T}\langle\phi, u\rangle_{q} d t\right| & =\frac{1}{2 \pi}\left|\int_{-\infty}^{+\infty}\left\langle\hat{\phi}(\omega), \hat{v}_{T}(\omega)\right\rangle_{q} d t\right| \leq \frac{1}{2 \varepsilon}\|\phi\|_{1 / k, q}^{2}+C_{2} \frac{\varepsilon}{2}\|\nabla u\|_{k, T}^{2}, \\
\left|\int_{0}^{T}\langle f, u\rangle d t\right| & =\frac{1}{2 \pi}\left|\int_{-\infty}^{+\infty}\left\langle\hat{f}(\omega), \hat{v}_{T}(\omega)\right\rangle d t\right| \leq \frac{1}{2 \varepsilon}\|f\|_{1 / k}^{2}+C_{3} \frac{\varepsilon}{2}\|\nabla u\|_{k, T}^{2}
\end{aligned}
$$

Thus we obtain

$$
\frac{\alpha_{0}}{2}\|u(T)\|^{2}+\mu\|\nabla u\|_{k, T}^{2} \leq \frac{\alpha_{0}}{2}\left\|u_{0}\right\|^{2}+\frac{1}{2 \varepsilon} C^{\prime}\left(\mathbf{Q}_{0}, f, \phi\right)+\int_{0}^{T}\left\|\alpha^{\prime} u_{0}\right\|\|u\| d t
$$

with $\mu=1-\varepsilon\left(1+C_{2}+C_{3}\right) / 2>0$ and $C^{\prime}\left(\mathbf{Q}_{0}, f, \phi\right)=\left(\left\|\mathbf{Q}_{0}\right\|_{1 / k}^{2}+\|\| \|_{1 / k, q}^{2}+\right.$ $\mid\|f\|_{1 / k}^{2}$ ). Applying again Gronwall's generalized lemma, for any $T \in \mathbb{R}^{+}$it follows that

$$
\begin{gathered}
\|u(T)\| \leq\left(\left\|u_{0}\right\|^{2}+\frac{1}{\alpha_{0} \varepsilon} C^{\prime}\left(\mathbf{Q}_{0}, f, \phi\right)\right)^{1 / 2}+\left\|u_{0}\right\| \int_{0}^{T} \frac{\left|\alpha^{\prime}\right|}{\alpha_{0}} d t, \\
\mu\|\nabla u\|_{k, T}^{2} \leq \frac{\alpha_{0}}{2}\left\|u_{0}\right\|^{2}+\frac{1}{2 \varepsilon} C^{\prime}\left(\mathbf{Q}_{0}, f, \phi\right)+\sup _{t \in(0, T)}\|u(t)\| \int_{0}^{T}\left\|\alpha^{\prime} u_{0}\right\| d t .
\end{gathered}
$$

Thus taking the limit as $T \rightarrow \infty$ and taking account of Poincaré's inequality, the theorem is proved.

REMARK 4.3. If $\alpha^{\prime}(0)>0$, we can control the norm of the solution in $L^{2}\left(\mathbb{R}^{+}, L^{2}(\Omega)\right)$. In fact, by substituting Poincaré's inequality into (4.2),

$$
\left\|\hat{v}_{T}(\omega)\right\| \leq 2 C(\omega)\left\|\nabla \hat{v}_{T}(\omega)\right\| \text { with } C(\omega)>0 \forall \omega \in \mathbb{R}
$$

and by setting $\beta(\omega)=\omega \hat{\alpha}_{\mathrm{s}}^{\prime}(\omega)+C(\omega) \hat{k}_{\mathrm{c}}^{\prime}(\omega)>0\left(\right.$ and $\left.\lim _{\omega \rightarrow \infty} \beta(\omega)=\alpha^{\prime}(0)>0\right)$, we see that

$$
\frac{1}{2 \pi} \int_{-\infty}^{+\infty} \beta(\omega)\left\|\hat{v}_{T}(\omega)\right\|^{2} d \omega \geq \frac{\beta}{2 \pi} \int_{-\infty}^{+\infty}\left\|\hat{v}_{T}(\omega)\right\|^{2} d \omega=\beta \int_{0}^{T}\|u(t)\|^{2} d t,
$$

where $\beta=\inf _{\omega \in \mathbb{R}} \beta(\omega)>0$. Moreover, in this case we do not need the requirement $f=r-\dot{U}_{0} \in L_{1 / k}^{2}\left(\mathbb{R}^{+}, L^{2}(\Omega)\right)$.

5. Restrictions on the data. In the sequel (H.5)-(H.6), imposed on the data when $k_{0}=0$, will be considered in detail. As an introduction we remark that, as $k^{\prime} \in L^{1}$, $\hat{k}_{\mathrm{c}}^{\prime}$ is bounded, continuous on $\mathbb{R}^{+}$, and goes to 0 as $\omega \rightarrow \infty$. Then, by (2.5) $k^{\prime}$ is a positive definite kernel, that is (see [20]),

$$
\int_{0}^{T} \int_{0}^{t} k^{\prime}(t-s) u(s) u(t) d s d t>0 \quad u \neq \equiv \quad \forall T \in \mathbb{R}^{+}
$$

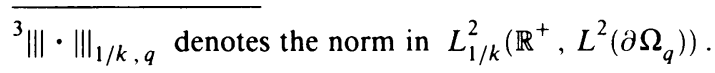


and, moreover, $k^{\prime}(0)=\frac{1}{\pi} \int_{0}^{+\infty} \hat{k}_{\mathrm{c}}^{\prime}(\omega) d \omega$; so $k^{\prime}(0)$ is finite and positive if and only if $\hat{k}_{\mathrm{c}}^{\prime} \in L^{1}$. Finally we recall (see [20]) that $k^{\prime}$ is a strongly positive definite kernel if $k^{\prime}(s)-\eta e^{-s}$ is a positive definite kernel for some $\eta>0$. It has been shown that if $k^{\prime} \in L^{1}$ then $k^{\prime}$ is strongly positive definite if and only if

$$
\hat{k}_{\mathrm{c}}^{\prime}(\omega) \geq \frac{\eta}{1+\omega^{2}}, \quad \eta>0, \forall \omega \in[0, \infty)
$$

In order to examine some properties of the functional spaces $L_{1 / k}^{2}\left(\mathbb{R}^{+}, V\right)$, where $V$ is $L^{2}(\Omega)$ or $L^{2}\left(\partial \Omega_{q}\right)$, we distinguish three cases:

1. $k^{\prime}$ is singular at the origin $\left(\hat{k}_{\mathrm{c}}^{\prime} \notin L^{1}\right)$ and

$$
\exists \alpha \leq 1, \gamma>0, \bar{\omega} \text { such that } \hat{k}_{\mathrm{c}}^{\prime}(\omega) \geq \frac{\gamma}{\omega^{\alpha}} \forall \omega>\bar{\omega} .
$$

By the continuity of $\hat{k}_{\mathrm{c}}^{\prime}$ and (2.5), there exists a positive constant $\varepsilon_{\bar{\omega}}$ such that $\hat{k}_{\mathrm{c}}^{\prime}(\omega) \geq \varepsilon_{\bar{\omega}}>0 \forall \omega \in[0, \bar{\omega}]$, and letting $\eta=\min \left\{\gamma, \varepsilon_{\bar{\omega}}\right\}>0$ we get

$$
\hat{k}_{\mathrm{c}}^{\prime}(\omega) \geq \frac{\eta}{1+\omega^{\alpha}} \quad \forall \omega \in[0, \infty) .
$$

THEOREM 5.1. When (5.2) holds there exists a real index $s \in(0,1 / 2]$ such that if $\mathbf{Q}_{0} \in H^{s}\left(\mathbb{R}^{+}, L^{2}(\Omega)\right)$ and $\phi \in H^{s}\left(\mathbb{R}^{+}, L^{2}\left(\partial \Omega_{q}\right)\right)$ then (H5)-(H6) are satisfied.

Proof. Let $f$ belong to $H^{s}\left(\mathbb{R}^{+}, V\right), 0<s \leq 1 / 2$, where $V$ is $L^{2}(\Omega)$ or $L^{2}\left(\partial \Omega_{q}\right)$ and $\|\cdot\|$ denotes the norm on $V$, and let $\|\cdot\|_{H^{s}}^{2}$ denote the norm on $H^{s}\left(\mathbb{R}^{+}, V\right)$. Then, by (5.3)

$$
\begin{aligned}
\|f\|_{1 / k}^{2} & =\frac{1}{2 \pi} \int_{-\infty}^{+\infty}\left(\hat{k}_{\mathrm{c}}^{\prime}(\omega)\right)^{-1}\|\hat{f}(\omega)\|^{2} d \omega \\
& \leq \frac{2}{\pi} \int_{-\infty}^{+\infty}\left(1+\omega^{2 s}\right) \eta^{-1}\|\hat{f}(\omega)\|^{2} d \omega=\eta^{-1}\|f\|_{H^{s}}^{2} .
\end{aligned}
$$

For instance let us consider a singularity of "log" type, i.e., $k^{\prime}(s)=-\beta \log s \chi_{\varepsilon}(s)+$ $\left(1-\chi_{\varepsilon}(s)\right) k^{\prime}(s)$ where

$$
\chi_{\varepsilon}(s)=\left\{\begin{array}{ll}
1 & s \leq \varepsilon, \\
0 & s>\varepsilon,
\end{array} \quad \text { and } \quad \beta=-\frac{k^{\prime}(\varepsilon)}{\log \varepsilon} .\right.
$$

In this case (5.2) holds with $\alpha=1$ and

$$
\begin{aligned}
\hat{k}_{\mathrm{c}}^{\prime}(\omega) & =\int_{0}^{\infty}\left[-\beta \log s \chi_{\varepsilon}(s)+\left(1-\chi_{\varepsilon}(s)\right) k^{\prime}(s)\right] \cos \omega s d s \\
& =-\int_{0}^{\infty}\left[\frac{\beta \chi_{\varepsilon}(s)}{s}-\left(1-\chi_{\varepsilon}(s)\right) k^{\prime \prime}(s)\right] \frac{\sin \omega s}{\omega} d s .
\end{aligned}
$$

Taking into account that $\hat{k}_{\mathrm{c}}^{\prime}(\omega) \sim O\left(\omega^{-1}\right)$ as $\omega \rightarrow \infty$, there exist $\gamma \geq 0$ and $\bar{\omega}$ such that

$$
\hat{k}_{\mathrm{c}}^{\prime}(\omega) \leq \frac{\gamma}{1+\omega} \quad \forall \omega>\bar{\omega}
$$


On the other hand, there is a positive constant $M$ such that $\hat{k}_{\mathrm{c}}^{\prime}(\omega) \leq M$ and in particular $\hat{k}_{\mathrm{c}}^{\prime}(\omega) \leq M(1+\bar{\omega}) /(1+\omega), \forall \omega \in[0, \bar{\omega}]$. So letting $\zeta=$ $\max \{\gamma, M(1+\bar{\omega})\}$ it follows that

$$
\hat{k}_{\mathrm{c}}^{\prime}(\omega) \leq \frac{\zeta}{1+\omega} \quad \forall \omega \in[0, \infty) .
$$

Corollary 5.1. If $k^{\prime}$ has a singularity of "log" type at $t=0$, then (H5)-(H6) hold if and only if $Q_{0} \in H^{s}\left(\mathbb{R}^{+}, L^{2}(\Omega)\right)$ and $\phi \in H^{s}\left(\mathbb{R}^{+}, L^{2}\left(\partial \Omega_{q}\right)\right)$, with $s=1 / 2$.

In fact, by (5.3)-(5.4) we obtain

$$
\frac{1}{\zeta}\left\|\left|\cdot\left\|_{H^{1 / 2}} \leq\right\|\right| \cdot \mid\right\|_{1 / k} \leq \frac{1}{\eta}\|\cdot\|_{H^{1 / 2}} .
$$

2. $k^{\prime}(0)<\infty$. By an analogous procedure we can establish the following:

THEOREM 5.3. If $k^{\prime}(0)<\infty$ then there exists a positive constant $s_{1} \geq 1 / 2$ such that (H5)-(H6) imply $\mathbf{Q}_{0} \in H^{s_{1}}\left(\mathbb{R}^{+}, L^{2}(\Omega)\right)$ and $\phi \in H^{s_{1}}\left(\mathbb{R}^{+}, L^{2}\left(\partial \Omega_{q}\right)\right)$.

Proof. As $\hat{k}_{\mathrm{c}}^{\prime} \in L^{1}$ there exist $s_{1} \geq 1 / 2, \gamma \geq 0$, and $\bar{\omega}$ such that

$$
\hat{k}_{\mathrm{c}}^{\prime}(\omega) \leq \gamma\left(1+\omega^{2 s_{1}}\right)^{-1} \quad \forall \omega>\bar{\omega}
$$

and, as before, the inequality $\hat{k}_{\mathrm{c}}^{\prime}(\omega) \leq M\left(1+\bar{\omega}^{2 s_{1}}\right)\left(1+\omega^{2 s_{1}}\right)^{-1}, \forall \omega \in[0, \bar{\omega}]$, holds. Therefore, if $\zeta=\max \left\{\gamma, M\left(1+\bar{\omega}^{2 s_{1}}\right)\right\}$ then it follows that $\hat{k}_{\mathrm{c}}^{\prime}(\omega) \leq \zeta\left(1+\omega^{2 s_{1}}\right)^{-1}$, $\forall \omega \in[0, \infty)$ and the conclusion follows by comparing the norms as in the previous theorem.

3. $k^{\prime}$ is strongly positive definite.

THEOREM 5.4. If $k^{\prime}(0)<\infty$ and $k^{\prime}$ is strongly positive definite, then there exists a positive constant $s_{2} \leq 1$ such that if $\mathbf{Q}_{0} \in H^{s_{2}}\left(\mathbb{R}^{+}, L^{2}(\Omega)\right)$ and $\phi \in H^{s_{2}}\left(\mathbb{R}^{+}, L^{2}\left(\partial \Omega_{q}\right)\right)$ then (H5)-(H6) hold.

Proof. In view of (5.1) we can find $s_{2} \leq 1$ and $\eta>0$ so that (5.3) is satisfied for $\alpha=2 s_{2}$. The conclusion follows by comparing the norms as in previous theorems.

Finally, we suggest another condition on the initial history that permits the conclusion in Theorem 4.2 to be true.

THEOREM 5.5. The conclusion of Theorem 4.2 is valid if $(\mathrm{H} 5)$ is replaced by

$$
\nabla u_{0} \in L_{k}^{2}\left(\mathbb{R}^{-}, L^{2}(\Omega)\right) \text {. }
$$

Proof. Consider

$$
v_{0}(\mathbf{x}, t)= \begin{cases}u_{0}(\mathbf{x}, t), & t \leq 0 \\ 0, & t>0\end{cases}
$$

so that $\mathbf{Q}_{0}$ may be represented as a convolution product $\mathbf{Q}_{0}=k^{\prime} *_{\mathrm{F}} \nabla v_{0}$ for every $t>0$. Let $k^{\prime}$ be the even-extension on $\mathbb{R}$ of the kernel, namely $k^{\prime}(s)=k^{\prime}(-s)$, and set

$$
\widehat{\mathbf{Q}}_{\mathrm{e}}(\mathbf{x}, \omega)=\int_{-\infty}^{+\infty}\left(k^{\prime} *_{\mathrm{F}} \nabla v_{0}\right)(\mathbf{x}, t) e^{-i \omega t} d t=2 \hat{k}_{\mathrm{c}}^{\prime}(\omega) \nabla \hat{v}_{0}(\mathbf{x}, \omega)
$$


Thus inequality (4.4) is valid even if $\left\|\mathbf{Q}_{0}\right\|_{1 / k}^{2}$ is replaced by

$$
\begin{aligned}
\left\|\mathbf{Q}_{\mathrm{e}}\right\|_{1 / k}^{2} & =\frac{1}{\pi} \int_{0}^{+\infty}\left(\hat{k}_{\mathrm{c}}^{\prime}(\omega)\right)^{-1}\left\|\widehat{\mathbf{Q}}_{\mathrm{e}}(\omega)\right\|^{2} d \omega \\
& =\frac{4}{\pi} \int_{0}^{+\infty} \hat{k}_{\mathrm{c}}^{\prime}(\omega)\left\|\nabla \hat{v}_{0}(\omega)\right\|^{2} d \omega \\
& =2\left\|\nabla u_{0}\right\|_{k}^{2} .
\end{aligned}
$$

Added in proof. Although Theorem 3.1 and Theorem 4.1 state existence and uniqueness in $L^{2}\left(\mathbb{R}^{+}, V_{0}\right)$, this kind of result is not crucial to prove stability. Indeed, one can build Theorem 3.2 and Theorem 4.2 starting from the weaker condition $u \in L^{2}\left(0, T ; V_{0}\right)$ that easily follows from Lemma 3.1 .

Acknowledgment. This work has been performed under the auspices of G.N.F.M.C.N.R. and partially supported by Italian M.U.R.S.T. through the $40 \%$ project "Problemi di evoluzione nei fluidi e e nei solidi".

\section{REFERENCES}

[1] B. D. Coleman and V. J. Mizel, Thermodynamics and departures from Fourier's law of heat conduction, Arch. Rational Mech. Anal. 13 (1963), 245-261

[2] B. D. Coleman and V. J. Mizel, Norms and semi-groups in the theory of fading memory, Arch. Rational Mech. Anal. 23 (1966), 87-123

[3] B. D. Coleman and M. E. Gurtin, Equipresence and constitutive equations for rigid heat conductors, Z. Angew. Math. Phys. 18 (1967), 199-208

[4] M. E. Gurtin, On the thermodynamics of materials with memory, Arch. Rational Mech. Anal. 28 (1968), 40-50

[5] M. E. Gurtin and A. C. Pipkin, A general theory of heat conduction with finite wave speeds, Arch. Rational Mech. Anal. 31 (1968), 113-126

[6] W. A. Day and M. E. Gurtin, On the symmetry of conductivity tensor and other restrictions in the nonlinear theory of heat conduction, Arch. Rational Mech. Anal. 33 (1968), 26-32

[7] J. W. Nunziato, On heat conduction in materials with memory, Quart. Appl. Math. 29 (1971), $187-204$

[8] W. A. Day, The thermodynamics of simple materials with fading memory, Springer, Berlin, 1972

[9] B. D. Coleman and D. R. Owen, A mathematical foundation for thermodynamics, Arch. Rational Mech. Anal. 54 (1974), 1-104

[10] F. Treves, Basic Linear Partial Differential Equations, Academic Press, New York, 1975

[11] R. K. Miller, An integrodifferential equation for rigid heat conductors with memory, J. Math. Anal. Appl. 66 (1978), 313-332

[12] V. L. Kolpashchikov and A. I. Shnip, Linear thermodynamic theory of heat conduction with memory, J. Engrg. Phys. 46 (1984), 732-739

[13] G. M. Troianiello, Elliptic Differential Equation and Obstacle Problems, Plenum Press, New York and London, 1987

[14] M. Fabrizio and B. Lazzari, On the stability of linear viscoelastic fluids, Differential Integral Equations, to appear

[15] G. Gentili, Dissipativity conditions and variational principles for the heat flux equation with memory, Differential Integral Equations 4 (1991), 977-989

[16] C. Giorgi, Alcune conseguenze delle restrizioni termodinamiche per mezzi viscoelastici lineari, Quad. Sem. Mat. Univ. Brescia, 6/91

[17] C. Baiocchi, Soluzioni ordinarie e generalizzate del problema di Cauchy per equazioni differenziali astratte lineari del II ordine in spazi di Hilbert, Ric. Mat. 16 (1967), 27-95 
[18] M. Fabrizio and A. Morro, On uniqueness in linear viscoelasticity: A family of counterexamples, Quart. Appl. Math. 45 (1987), 321-325

[19] H. Grabmueller, On linear theory of heat conduction in materials with memory, Proc. Roy. Soc. Edinburgh, A-76 (1976-1977), 119-137

[20] M. Renardy, W. J. Hrusa, and J. A. Nohel, Mathematical problems in viscoelasticity, Pitman monographs and surveys in pure and applied mathematics, Longman, Essex, 1987 